\title{
PUBLIC HEALTH INFORMATICS
}

\section{Eva to the rescue}

Nature https://doi.org/10.1038/s41586-021-04014-z (2021)

Different countries have implemented various screening strategies at their borders to control the spread of COVID-19, especially from asymptomatic travelers. Most of these strategies are based on population-level epidemiological metrics, such as number of cases and number of deaths per capita; for instance, a country may require a negative test or quarantine upon the arrival of a traveler, or simply forbid their entrance at the border, if the location from which they are coming has had a high number of COVID-19related cases or deaths. These metrics are, however, affected by issues such as underreporting and testing biases, which in turn can affect their effective use for screening travelers. In a recent work, Kimon Drakopoulos and colleagues report the design and implementation of a fully algorithmic approach, not dependent on the aforementioned metrics, for targeted COVID-19 screening.

The main intuition of the proposed algorithmic approach, named Eva, is to use demographic features that are collected on an entry form (examples include region, age, and gender) to generate traveler types, and then to generate prevalence statistics based on these types. The system leverages the prevalence estimates to select travelers for group testing, instead of using general population-level metrics to adopt screening strategies solely based on country of origin. Implementation-wise, the traveler types are created using LASSO regression, and the statistics are computed using the Bayes method. It is worth noting, however, that COVID-19 prevalence can change quickly and abruptly, and at different rates across different traveler types. Therefore, in order to keep the prevalence estimates as accurate and updated as possible, the authors formulate their problem as a multi-armed bandit problem (a classic reinforcement learning problem): the system maximizes the number of infected asymptomatic travelers identified (exploitation phase of the algorithm), while strategically allocating tests to traveler types for which a more precise estimate is needed (exploration phase of the algorithm).

Eva was deployed from August to November 2020 across all points of entry within Greece. In their analysis, the authors showed that random testing (which was Greece's initial screening proposal), when compared against the proposed target testing approach, would have only identified about half of the infected travelers that Eva identified. In addition, Eva identified between 1.25 and 1.45 times more infections than when using target testing based on population-level metrics. As a matter of fact, by making use of state-of-the-art machine learning algorithms (including gradient boosting machine), the authors demonstrated that population-level metrics have almost no predictive power for detecting prevalence among asymptomatic travelers. All in all, Eva has the potential to help authorities to make a more informed decision at the borders, which can be beneficial for mitigating the spread of SARS-CoV-2 and other infectious diseases in the future.

\section{Fernando Chirigati}

Published online: 8 October 2021 https://doi.org/10.1038/s43588-021-00147-3 